\title{
Evaluation of different InSAR multi- baseline construction methods over a dam in southern Italy
}

Claudia Pipitone, Antonino Maltese, Gino Dardanelli, Fulvio Capodici, Jan-Peter Muller, et al.

Claudia Pipitone, Antonino Maltese, Gino Dardanelli, Fulvio Capodici, JanPeter Muller, Goffredo La Loggia, "Evaluation of different InSAR multibaseline construction methods over a dam in southern Italy," Proc. SPIE 10783, Remote Sensing for Agriculture, Ecosystems, and Hydrology XX, 107831A (10 October 2018); doi: 10.1117/12.2326909

SPIE. Event: SPIE Remote Sensing, 2018, Berlin, Germany 


\title{
Evaluation of different InSAR Multi-Baseline Construction methods over a dam in southern Italy
}

\author{
Claudia Pipitone ${ }^{1}$, Antonino Maltese ${ }^{1}$, Gino Dardanelli ${ }^{1}$, Fulvio Capodici ${ }^{1}$, Jan-Peter Muller ${ }^{2}$, \\ Goffredo La Loggia ${ }^{1}$
}

\author{
${ }^{1}$ Dipartimento di Ingegneria Civile Ambientale, Aerospaziale, dei Materiali, Università degli Studi di Palermo, Bld. 8, \\ Viale delle Scienze, Palermo, Italy. \\ ${ }^{2}$ Mullard Space Science Laboratory (MSSL), Department of Space \& Climate Physics, University College London \\ (UCL), Holmbury St Mary, RH5 6NT, United Kingdom.
}

\begin{abstract}
Monitoring dam displacements using different techniques allows an evaluation of their structural behaviour over time. In this study, dam displacements (for the Castello dam, Agrigento, Italy) have been investigated using different Interferometric Synthetic Aperture Radar (InSAR) techniques exploiting a freely available dataset from the EU Copernicus Sentinel-1 SAR built by the European Space Agency (ESA). The dataset includes Sentinel 1A (S1A) images acquired in dual-polarization and Interferometric Wide (IW) swath using the Terrain Observation with Progressive Scans SAR (TOPSAR) mode. Three main Multi-Baseline Construction methods based on the identification of Persistent Scatterers (PS) have been tested, within a scene including an extra-urban area surrounding the dam. The evaluation of the best strategy is carried out over few images (7) with a constant acquisition time-span of 12 days, except for the first image, acquired 24 days before the next one. Three different multi-baseline construction methods have been investigated in this preliminary research to test the capability of these InSAR techniques in finding a time series of displacements with high accuracy in extra-urban areas also. The star graph results in displacement appear to be more in agreement with GNSS measurements than other techniques.
\end{abstract}

Keywords: Satellite Interferometry, Persistent Scatterers, Sentinel-1, TOPSAR, Multi-Baseline, displacements, dam

*claudia.pipitone02@unipa.it; phone: +39 09123896224

Remote Sensing for Agriculture, Ecosystems, and Hydrology XX, edited by Christopher M. U. Neale, Antonino Maltese, Proc. of SPIE Vol. 10783, 107831A · (c) 2018 SPIE · CCC code: 0277-786X/18/\$18 · doi: 10.1117/12.2326909 


\section{INTRODUCTION AND STATE OF THE ART}

The displacements of the Castello dam have been investigated over time using different satellite techniques to analyse the behaviour of a strategic structure, occasionally monitored with traditional instruments. The aim is to use the Sentinel1 dataset to detect both the deformations with the same accuracy of traditional instruments and to relate deformations with water levels determined by classifying the same images.

A first preliminary analysis pointed out the correlation between water levels and displacements over a period of three months, using optical remote sensing and Global Navigation Satellite System (GNSS) ${ }^{1}$. Then, the GNSS displacements were compared with existing models based on a Finite Element Method (FEM) ${ }^{2}$. The time series of GNSS displacements has been determined using a single baseline connecting the receiver on the dam and the Continuously Operating Reference Stations (CORS) of Agrigento, about 30 kilometers away. Then, a more consistent dataset based on remote sensing acquisitions (including both optical and SAR images) was chosen to analyse the water level' variations and their relationship with the displacements found in the middle section of the dam using GNSS $^{3}$. According to these results, two other approaches have been tested to retrieve the water levels using remote sensing datasets with different resolutions and under different conditions, including windy, shadowed, and clear images ${ }^{4}$.

In this work, the dam displacements are further investigated using the InSAR technique. The PS-InSAR (Permanent Scatters for Interferometry Synthetic Aperture Radar), originally introduced in $2001^{5}$ adopts natural targets with high reflectivity over time to analyze the ground motions or topographic changes over a stack of SAR images. The technique is considered a powerful method for building stability estimates and ground motion detection, especially in urban areas ${ }^{6-}$ ${ }^{7}$, but many works shown the capability of this method for dam monitoring ${ }^{8-10}$. In addition, several studies evaluated different InSAR strategies to increase the number of suitable points for motion detection ${ }^{11}$ also in extra-urban areas ${ }^{12}$.

In particular, to test the InSAR technique over extra-urban areas, different Multi-Baseline Construction methods have been tested and results compared in terms of coherence and displacements.

\section{MATERIALS}

The study area includes an embankment dam located in south Italy, previously analysed to detect the relationship between the displacements and the water levels involving GNSS and remote sensing (Figure 1). The dam is built of coarse-grained homogeneous alluvium from the valley and limestone and a seal coat of bituminous conglomerate. It is composed of an arc of $310 \mathrm{~m}$ length between two segments of 165 and $317 \mathrm{~m}$ on the right and left hydraulic sides, respectively. The material covering the left bank on the upstream side of the dam is made up of coarse-grained alluvial material standing on a stone plinth.

The dataset includes S1A images, acquired in ascending orbits in Single Look Complex (SLC) format. The SAR images in VV polarization have been processed over a year (2016) and, after preliminary results, selected images have been chosen to test the Multi-Baseline Construction methods.

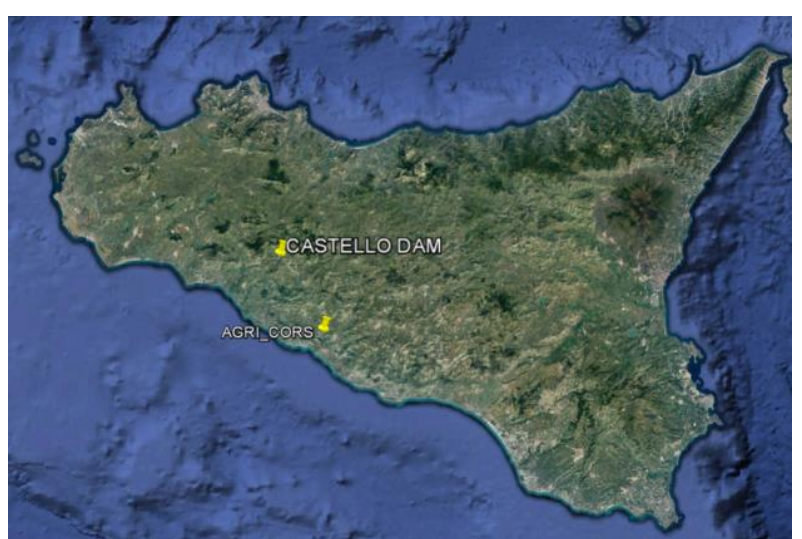

(a)

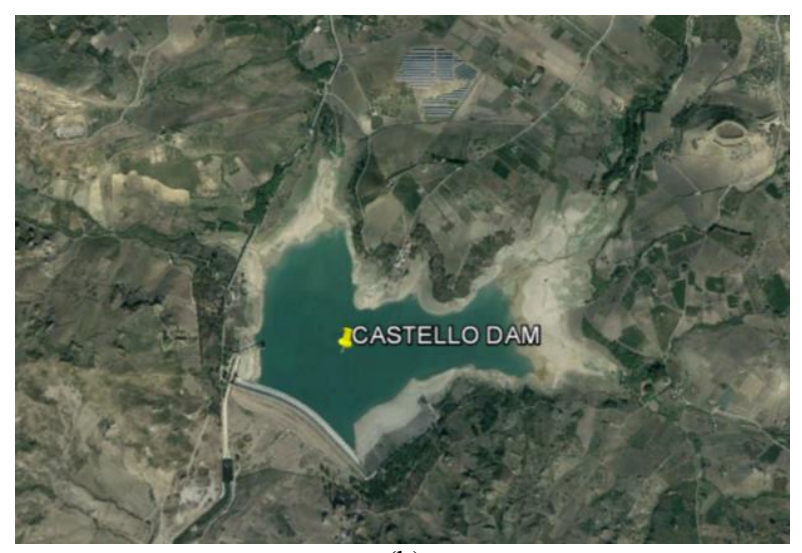

(b)

Figure 1. Overview of the study area in Sicily, Italy: (a) Castello dam (b) from Google Earth. 


\section{METHODS}

Sentinel-1 images acquired in ascending orbits in 2016 have been analyzed using the SARProZ software ${ }^{13} \odot$. The dataset was co-registered assuming as master reference an image centred in space and time. Based on the spatial and temporal baselines connecting the images, and on the computational cost for the processing, $n$ highly coherent images ( 7 in this case) were selected to test a more suitable processing method.

In this work, three different multi-baseline construction methods were tested: the classical Star Image graphs small baseline ${ }^{14}$; the Minimum Spanning Tree, MST ${ }^{11}$, and the Full graph.

The Star Image graph is typically used for PS analysis, every slave is connected to the master through a baseline ( $n-1$ connections, excluding the master-master connection), it minimizes the baseline in space and time.

The MST graph maximizes the average coherence to obtain best interferograms ( $n$ connections); connections of MST graph are more vertical than in the star graph meaning that smaller temporal baselines are achieved.

The full graph uses all available pairs connections $(n ! / 2(n-2) !, 22$ connections in this case), thus it is redundant compared to the previous two but generally requires a much higher computational cost. By using only few images and processing the data over a small area as in this test, not significant difference were found between the Multi-Baseline Construction methods in terms of computational time.

Based on different baselines and using a relative reference point in a stable location, the selected PS on the dam, will result in a displacement time series.

A preliminary geocoding is needed after co-registration; for this purpose, the digital surface model available from the Shuttle Radar Topography Mission (SRTM) has been used; also, the reflectivity map (Figure 2d) and the amplitude stability index have been computed. Then, a reference point is selected to evaluate the interferometric phase of all PSs within the study area, after selecting a threshold in terms of amplitude. The reference point, characterized by high reflectivity, has been chosen in the same position for the three analyses, an area considered stable close to the dam.

After preliminary InSAR processing, an Atmospheric Phase Screen (APS) is estimated using the inverted residuals; thus, the PS to be analysed are selected using a threshold for the Amplitude Stability Index (ASI). Using more redundant interferograms, as in the case of the multi-master approaches, the coherence are used as weights during the estimation of APS and PS, while no weights have been applied using the traditional star-graph processing. Also, a spatial filtering has been applied to enhance the signal of the interferometric phase of the targets included within the scene to detect the displacements after unwrapping.

Later, after the geocoding from SAR coordinates, a time series of deformations in the Line of Sight (LOS) of the satellite has been analysed.

\section{RESULTS}

The preliminary results for different graphs are available from 7 selected images over a small area surrounding the reservoir. The displacements of natural targets on the dam have been selected and analysed to evaluate a more suitable strategy. 


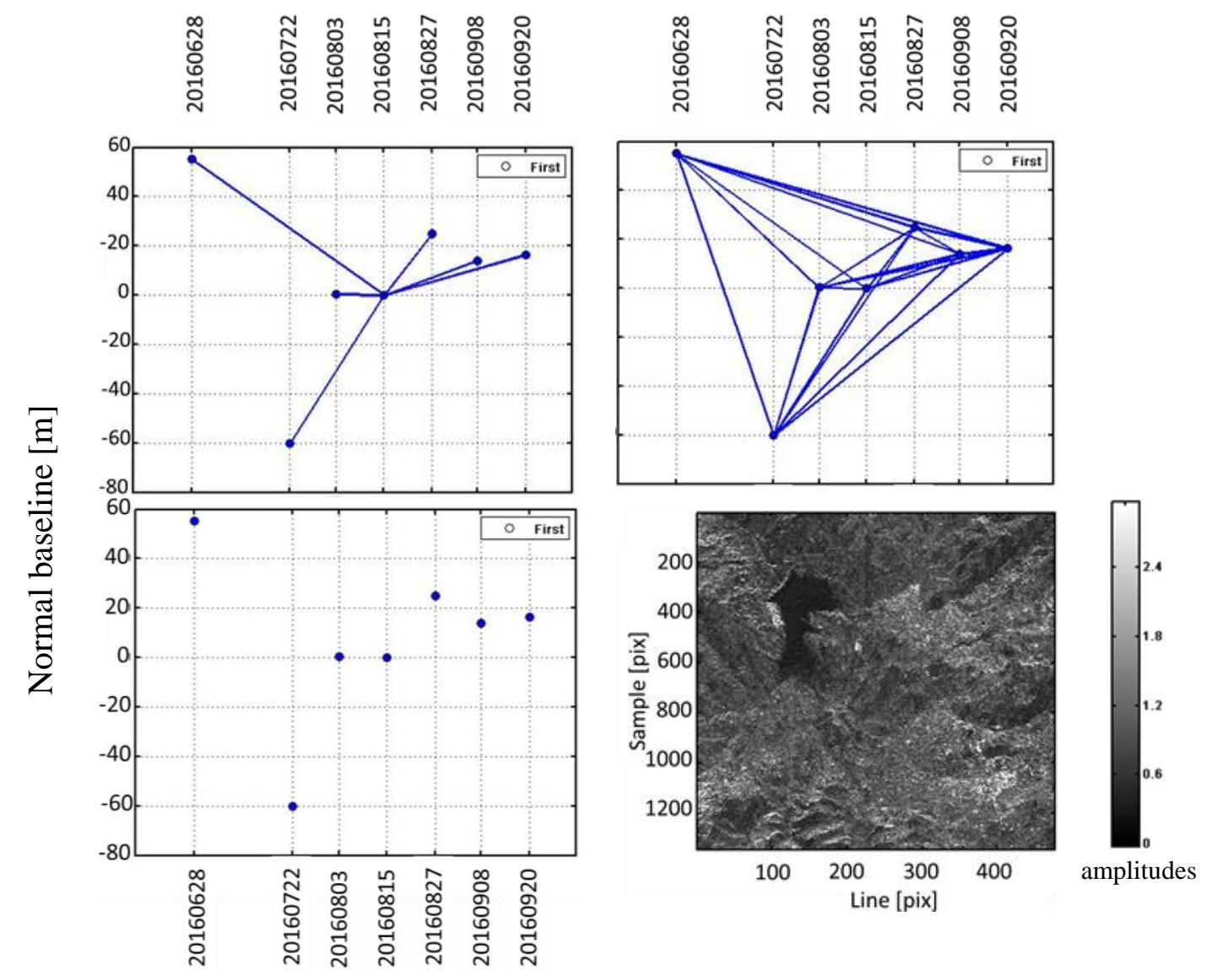

Figure 2. Baseline extraction for multi-image processing: star graph (top left panel), MST graph (lower left) and full graph (top right). In the lower right panel, a reflectivity map is shown in SAR coordinates.

Figure 2 shows the graph images before InSAR processing. In the star graph (top left panel) each image represented by one point interferes only with the master image (15/08/2016), chosen in a barycentre position; in the MST graph (lower left panel) the connections are not yet available because the best combination of interferograms maximizing the coherence, reducing the decorrelation, is obtained after the interferograms generation.

In the full graph in the upper right panel, connections are reported for all available pairs. The processing based on this graph has generally a higher computational cost, especially over large areas or using several images. Even though the use of redundant connections could be useful to increase the accuracy of the deformation evaluations (Figure 4), anyway; in this case, the images adopted are few, and not all the available images are highly correlated.

The coherence of all the image pairs is shown in Figure 3 for each technique. The reflectivity map shows medium-high amplitudes over the dam itself, especially on the left hydraulic side (Figure 2d). The drawback was that coherent scatterers were not well distributed along the scene (since the area surrounding the dam was mainly vegetated). 


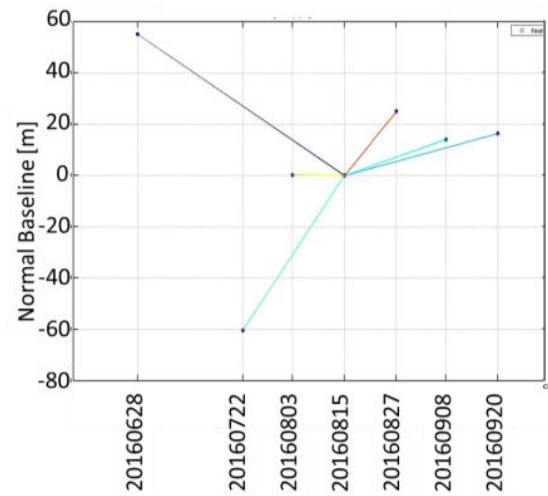

(a)

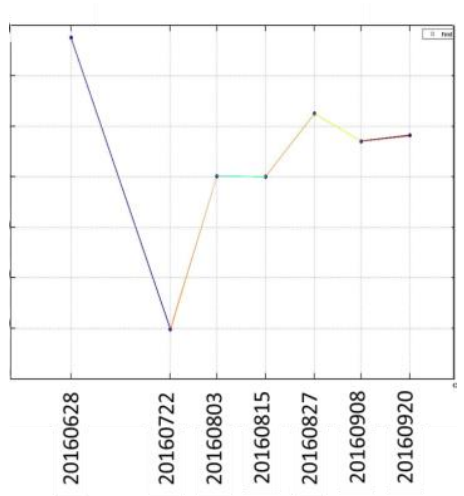

(b)

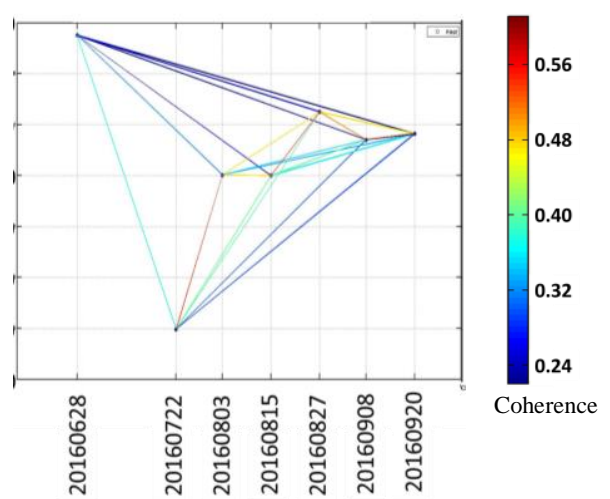

(c)

Figure 3. Coherence of the baselines using three different algorithms: a) star-graph, b) MST, c) full graph.

The interferograms processed using the full graph (Figure 4) are more clean when pairs are characterized by a small temporal baseline as expected. In this case, the time span is 12 days, except for the first two images ( 24 days). For the multi-master approaches, the spatial coherence was used to weight to generate the interferometric phase.

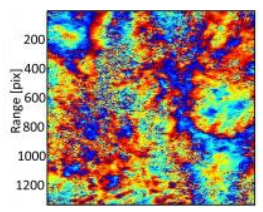

28/06-22/07

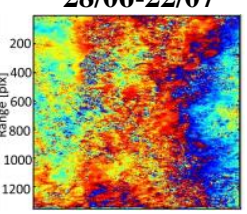

$22 / 07-15 / 08$

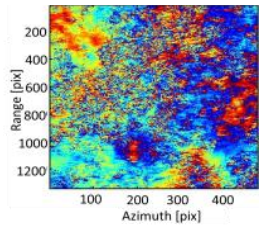

03/08-20/09

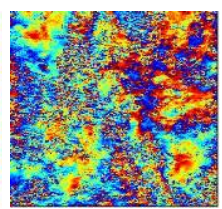

$28 / 06-03 / 08$

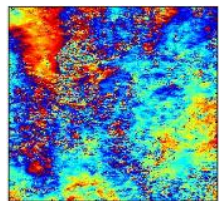

$22 / 07-27 / 08$

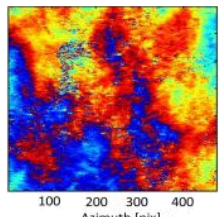

15/08-27/08

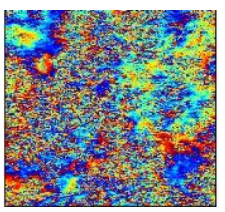

28/06-15/08

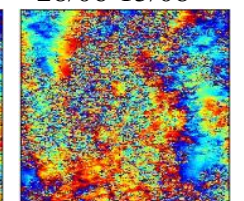

$22 / 07-08 / 09$

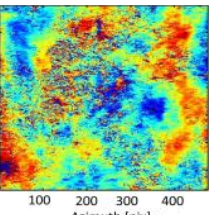

15/08-08/09
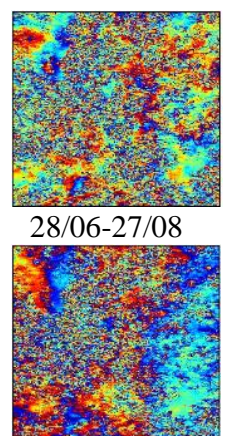

22/07-20/09

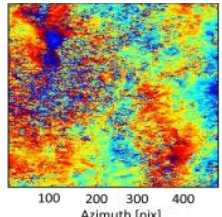

15/08-20/09

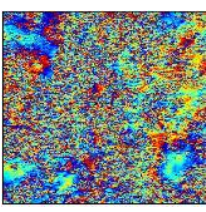

28/06-08/09

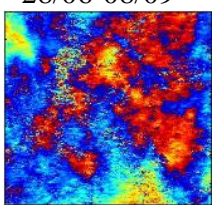

03/08-15/08

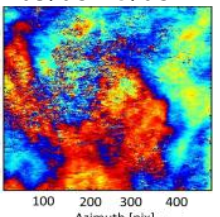

27/08-08/09

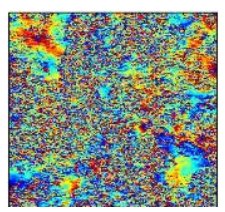

28/06-20/09

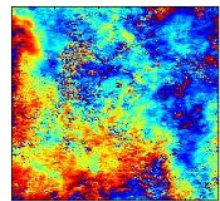

03/08-27/08

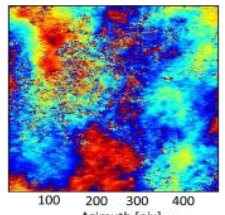

27/08-20/09
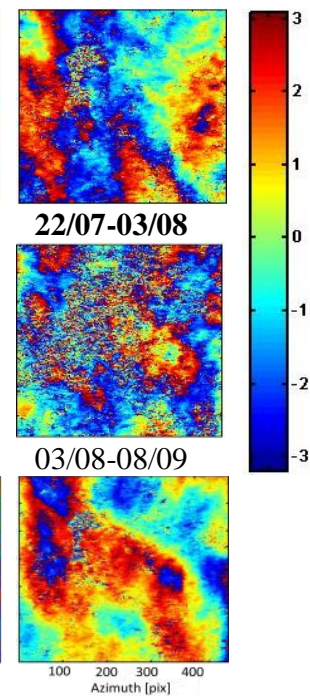

08/09-20/09

Figure 4. All available interferograms (full graph). In bold text, all the interferograms used in the MST graph are indicated.

After the APS estimate, the PS points have been retrieved over the study area and in particular over the dam, with three different multi-baseline construction methods. Using more interferograms and different combinations between the images, the points found on the dam show unexpected behavior. Indeed, the number of points over the dam decreases using all the connections (Figure 5b) as well as the coherence for both MST and full graphs (Figure 5, a and b). On the contrary, best results have been found using the star graph. Indeed, many PS points with high spatial and temporal coherence have been detected over the dam, especially on the left hydraulic side. In particular, more than half points detected over the scene have coherence higher than 0.8 , while using different approaches the points detected on the dam show low values of coherence. 


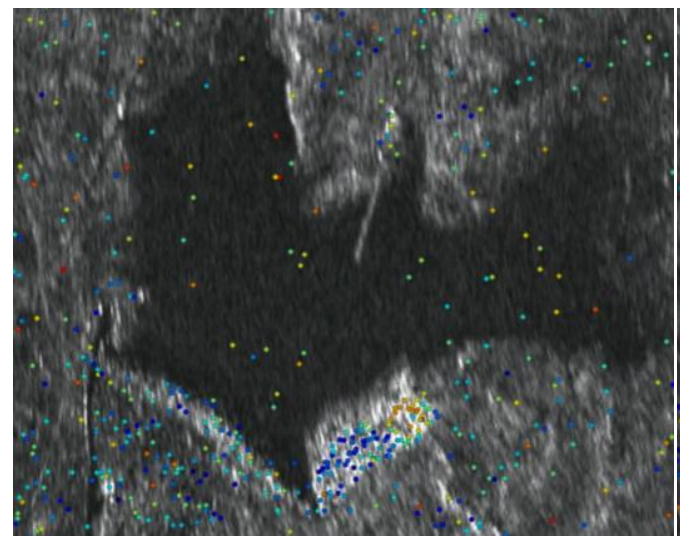

(a)

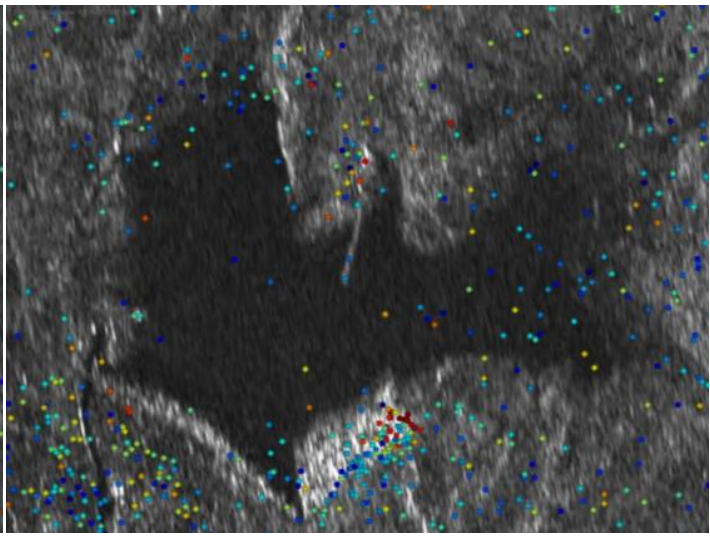

(b)

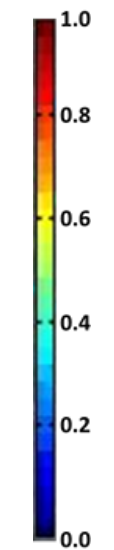

Coherence

Figure 5. Sparse points characterized by different values of temporal coherence over reflectivity map: a) MST and b) full graph.

The results agreed with the GNSS time series recorder in the period (April 2011-April 2012) ${ }^{3}$. In particular, the direction of LOS displacements are aligned with the one monitored via GNSS during the emptying period (Figure 6). Planimetric displacements will be quantified by processing more images both in ascending and descending orbits.
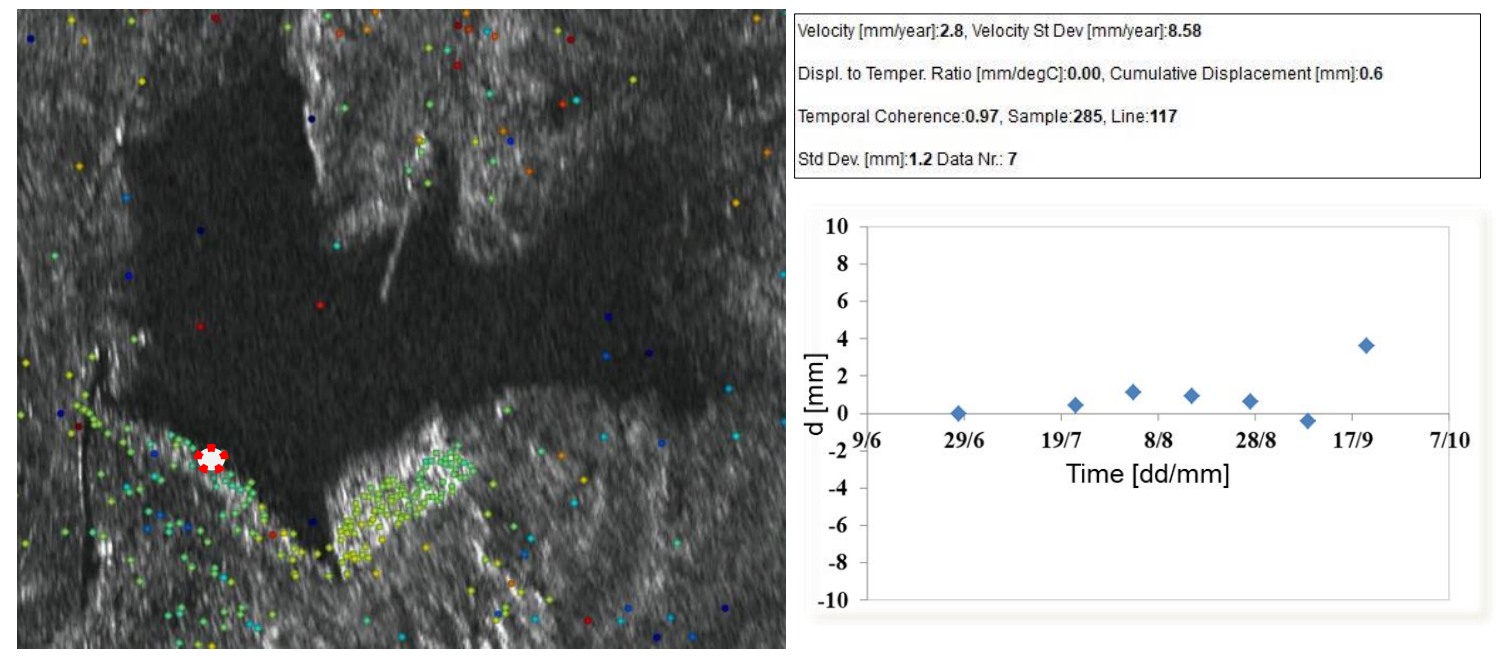

Figure 6. On the left panel, the deformation trend of points characterized by high spatial and temporal coherence is superimposed on an orthorectified reflectivity map. On the right, the time series displacements of a PS located over the dam (red circle on the reflectivity map).

An analysis of a more robust dataset (covering at least a year) is ongoing to analyse the displacements of the dam during the emptying and filling periods. The analysis of a larger dataset could characterize the relationship between displacements and water level fluctuations.

\section{CONCLUSIONS}

This preliminary study aimed to compare different strategies to evaluate the deformation fields over extra-urban areas where targets are not highly coherent and not well distributed around the scene. Indeed, the PS technique, using the 
traditional star-graph strategy, allows the detection of deformations of PS with a precision of $\sim 10 \mathrm{~mm}$, especially in urban areas. The study was conducted for 7 images with a nominal acquisition time-span of 12 days (except for the first two images separated by 24 days). Our analysis points out that when the temporal baseline between the pairs of images is small the interferograms are less noisy and also the accuracy of the results improves, otherwise when the temporal baseline increases the correlation is low, accuracies are worst. Best results in terms of displacements and expected behaviour of the dam during the emptying period have been found using the traditional PS method, involving the star graph for the images' network connections. Using the full graph, satisfactory results have not been found for this case study. Probably, using a more consistent dataset and more redundant interferograms, the results may have been evaluated. The use of the MST graph determined coherent interferograms but the results in terms of deformation trend estimation and displacements needs to be further investigated. Indeed, the characteristics of the points found using the multi-master approach does not seem satisfactory for this analysis in terms of coherence and deformation trend. Future work will exploit the use of different strategies used previously in urban areas, with a more consistent dataset, to highlight the differences in terms of accuracy of the methods in different cases.

\section{ACKNOWLEDGMENTS}

The authors would like to thank the European Space Agency (ESA) for providing the Sentinel-1A images. The authors wish to thank the Mullard Space Science Laboratory (MSSL), University College London (UCL) for hosting C. Pipitone as visiting scientist from September 2017 to February 2018. The software used in this work is SARProZ $\odot$, developed by D. Perissin. The authors are grateful to the developer also for his technical support.

\section{REFERENCES}

[1] Dardanelli, G., La Loggia, G., Perfetti, N., Capodici, F., Puccio, L., Maltese, A., "Monitoring displacements of an earthen dam using GNSS and remote sensing," Proc. SPIE 9239, 923928 (2014).

[2] Dardanelli, G. and Pipitone C., "Hydraulic models and finite elements for monitoring of an earth dam, by using GNSS techniques," Periodica Polytechnica Civil Engineering 61(3), 421-433 (2017).

[3] Pipitone, C., Maltese, A., Dardanelli, G., Lo Brutto, M., La Loggia, G., "Monitoring Water Surface and Level of a Reservoir Using Different Remote Sensing Approaches and Comparison with Dam Displacements Evaluated via GNSS," Remote Sensing 10(1), 71 (2018).

[4] Pipitone, C., Maltese, A., Dardanelli, G., Capodici, F., Lo Brutto, M., La Loggia, G., "Detection of a reservoir water level using shape similarity metrics," Proc. SPIE 10421, 104211L (2017).

[5] Ferretti A., Prati C., Rocca F., "Permanent scatterers in SAR interferometry," IEEE Transactions on Geoscience and Remote Sensing 39 (1), 8-20 (2001).

[6] Dixon, T. H., Amelung, F., Ferretti, A., Novali, F., Rocca, F., Dokka, R., Sella, G., Kim, S. W., Wdowinski, S., Whitman, D., "Subsidence and flooding in New Orleans," Nature 441, 587-588 (2006).

[7] Ng, A. H. M., Ge, L., Li, X., Zhang, K., "Monitoring ground deformation in Beijing, China with persistent scatterer SAR interferometry," Journal of Geodesy 86 (6), 375-392 (2012).

[8] Lazecký, M., Perissin, D., Lei, L., Qin, Y., Scaioni, M., "Plover Cove Dam Monitoring with Spaceborne InSAR Technique in Hong Kong," Proc. $2^{\text {nd }}$ JISDM, Nottingham, 9-10 September (2013).

[9] Tomás, R., Cano, M., García-Barba, J., Vicente, F., Herrera, G., Lopez-Sanchez, J. M., Mallorquí, J. J., "Monitoring an earthfill dam using differential SAR interferometry: La Pedrera dam, Alicante, Spain," Engineering Geology 157, 21-32 (2013).

[10] Lazecký, M., Perissin, D., Zhiying, W., Ling, L., \& Yuxiao, Q., “Observing Dam’s Movements with Spaceborne SAR Interferometry,” Engineering Geology for Society and Territory, Springer 5(3),131-136 (2015).

[11] Perissin, D.; Piantanida, R.; Piccagli, D.; Rocca, F., "Landslide in Dossena (BG): Comparison between interferometric techniques," Biogeosar 9, 1-7 (2007).

[12] Hooper, A., Segall, P., Zebker, H., "Persistent scatterer interferometric synthetic aperture radar for crustal deformation analysis, with application to Volcan Alcedo, Galapagos," Journal of Geophysical Research 112, B07407 (2007).

[13]Perissin, D., SARPROZ Manual, Copyright (c). http://www.sarproz.com/software-manual/ (2009-2017).

[14] Lanari, R., Mora, O., Manunta, M., Mallorquí, J. J., Berardino, P., Sansosti, E., "A small-baseline approach for investigating deformations on full-resolution differential SAR interferograms," IEEE Transaction on Geoscience and Remote Sensing 42(7), 1377-1386 (2004). 